\title{
Gradhiva
}

GRADHIV

Revue d'anthropologie et d'histoire des arts

$30 \mid 2019$

Précieux

\section{Axiologie du précieux. Essai de modélisation}

The Axiology of Preciousness. Some models

\section{Nathalie Heinich}

\section{(2) OpenEdition}

Journals

Édition électronique

URL : http://journals.openedition.org/gradhiva/4451

DOI : 10.4000/gradhiva.4451

ISSN : 1760-849X

\section{Éditeur}

Musée du quai Branly Jacques Chirac

\section{Édition imprimée}

Date de publication : 4 décembre 2019

Pagination : 92-107

ISBN : 978-2-35744-113-2

ISSN : 0764-8928

\section{Référence électronique}

Nathalie Heinich, «Axiologie du précieux. Essai de modélisation », Gradhiva [En ligne], 30 | 2019, mis en ligne le 31 mars 2021, consulté le 02 avril 2021. URL : http://journals.openedition.org/gradhiva/4451 ; DOI : https://doi.org/10.4000/gradhiva.4451 


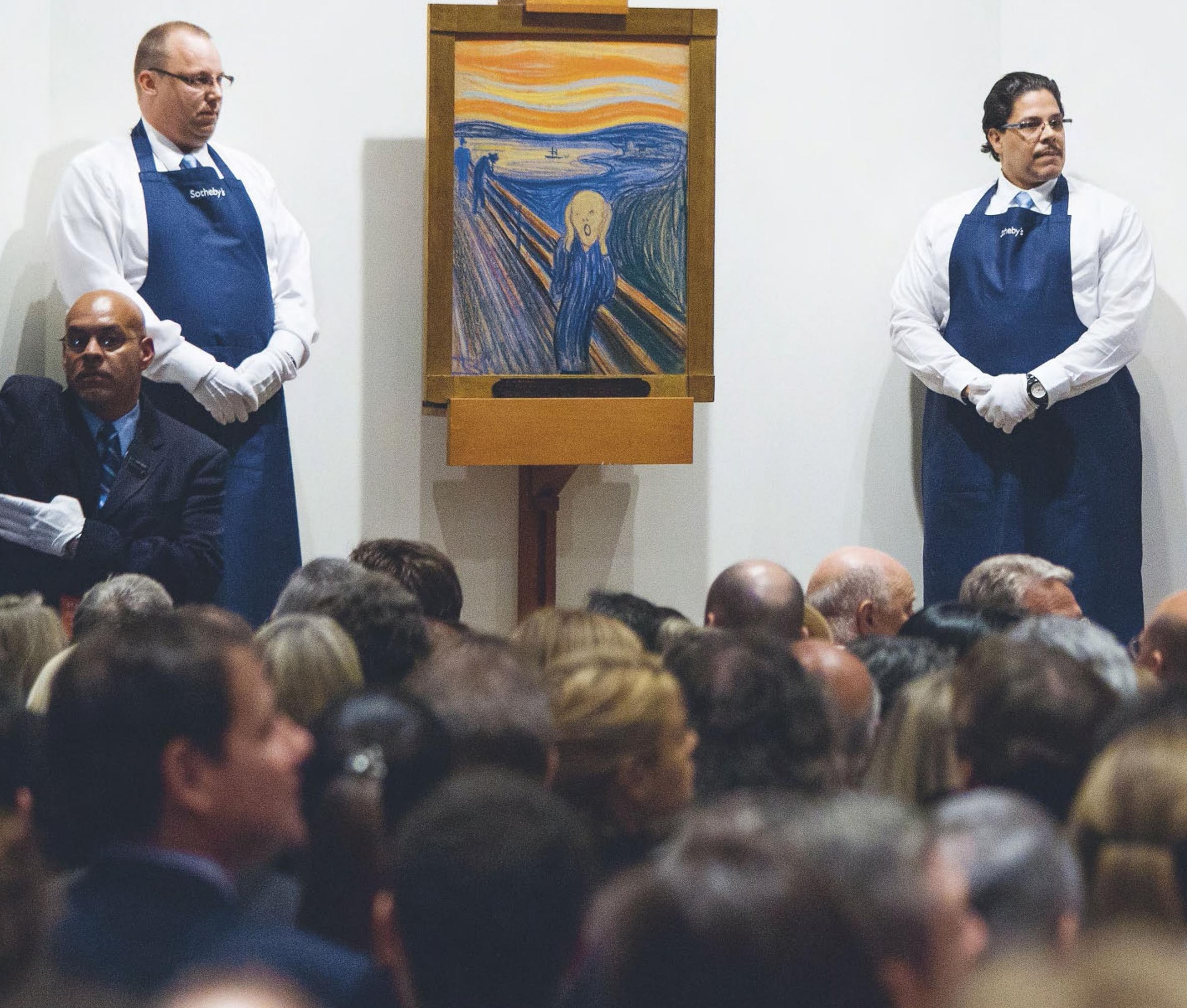


Axiologie

du précieux.

Essai de

modélisation.

Mots clefs:
évaluation, objets-personnes,

sacralisation, sociologie,

axiologique, valeurs

Nathalie

Heinich 
Cet article vise à caractériser le «précieux» à la lumière d'une modélisation des opérations d'attribution de valeur qui conduisent à une telle qualification, que ce soit par des mesures, des attachements ou des jugements, et qu'il s'agisse de choses, de personnes, d'actions ou d'états du monde. On y constatera la non-pertinence de la réduction économiste au «coûteux» pour définir le précieux, en même temps que l'importance des notions d'insubstituabilité, propre aux «objets-personnes» (les reliques, les fétiches, les œuvres d'art), et d'inaliénabilité, propre aux objets de patrimoine. Circulant de La Joconde au doudou, d'un tableau de Van Gogh à la robe de Marilyn ou à l'autographe de Johnny, I'on parcourra un répertoire de valeurs multiples: authenticité, beauté, plaisir, virtuosité, célébrité, spiritualité, affectivité, proximité, fonctionnalité, significativité, pérennité, rareté, universalité. E† l'on observera comment se déploient à propos du précieux les trois sens du mot «valeur»: la grandeur, le bien, le principe. Enfin, à la lumière de cet outil qu'est la «grammaire axiologique», nous serons en mesure de décider si et à quelles conditions la notion de «sacré» peut nous aider à penser la question du précieux.

1. Pour une bibliographie concernant les significations affectées au patrimoine, voir Heinich 2009a.

2. C'est ce que soutiennent Luc Boltanski et Arnaud Esquerre (2017).

3. Cette réflexion a été développée dans Heinich 2017a. Le lecteur pourra s'y reporter pour une présentation plus détaillée et pour la bibliographie de référence.

\section{LE PRÉCIEUX, DU GOÛTEUX AU SANS PRIX}

Partons du sens commun: le «précieux», c'est d'abord le coûteux, ce qui vaut très cher. Ce sont les pierres précieuses, les bijoux, les objets de luxe. Parfois, ce sont aussi des objets usuels, naguère sans valeur autre que d'usage mais qui sont devenus précieux parce qu'ils ont appartenu à un être hors du commun : des reliques donc, comme le clou de la Sainte Croix, la robe de Marilyn ou les chaussons de Noureev (Heinich 2012a). Parfois encore ce sont des œuvres d'art dont la valeur pécuniaire s'est envolée en salles des ventes en raison de la ǵrandeur reconnue à leur auteur - telle une toile de Van Gogh.

Cette toile se retrouve-t-elle dans un musée?

Elle en sera d'autant plus précieuse, au point d'attirer des foules de visiteurs - mais là, les choses se compliquent du fait qu'elle ne sera plus qualifiable de «coûteuse». En effet, une fois patrimonialisés les objets deviennent inaliénables ou, du moins, difficilement accessibles à la circulation marchande. Ils sont rendus à l'état de «trésor», que la génération actuelle hérite des générations précédentes et se doit de soustraire de l'échange et même du don, afin de le conserver pour les générations futures (Godelier 1996) - le patrimoine étant la forme occidentale, moderne, du trésor des sociétés traditionnelles 1 .

D'où la nécessité d'étendre la conception de sens commun au-delà de l'association spontanée entre le précieux et le coûteux, la valeur et le prix: contrairement à l'approche économiste, ce comble du précieux qu'est l'objet de patrimoine - cible de tous les soins, de toutes les attentions, de toutes les protections - est un objet «sans prix», échappant pour l'essentiel à l'évaluation monétaire. Certes, on peut objecter à cet arǵument de la valeur sans prix que même les objets de patrimoine sont affectés d'un prix à travers la valeur d'assurance ${ }^{2}$. Mais l'on peut objecter à cette objection que c'est là un prix présenté d'emblée comme fictif, et destiné à apprécier non pas la valeur de l'objet lui-même, mais le montant des compensations considérées comme acceptables en cas de perte ou d'endommagement. Il en va de même d'ailleurs des sommes accordées par les tribunaux aux familles victimes de la perte d'un proche: ces sommes ne correspondent pas au «prix» de la personne disparue, mais à la compensation financière pour sa perte (celle-ci étant d'ailleurs presque toujours jugée sans véritable équivalence avec celle-là).

L'on voit ainsi que l'extension de la catégorie du «précieux» à l'objet patrimonial constitue une première pierre dans le jardin des conceptions ordinaires, fortement imprégnées d'économisme: dès lors qu'un objet se trouve patrimonialisé et, corrélativement, considéré comme un objet précieux, sa valeur n'est pas mesurable par un prix ${ }^{3}$.

Pensons à présent à une autre catégorie d'objets à la fois précieux et sans prix: les doudous. La peluche du bébé est infiniment précieuse à ses yeux : il s'y accroche et hurle s'il le croit perdu. Car le doudou est un fétiche dans la mesure où, comme tout fétiche, il agit: il console et rassure le bébé (et l'on pourrait en dire 


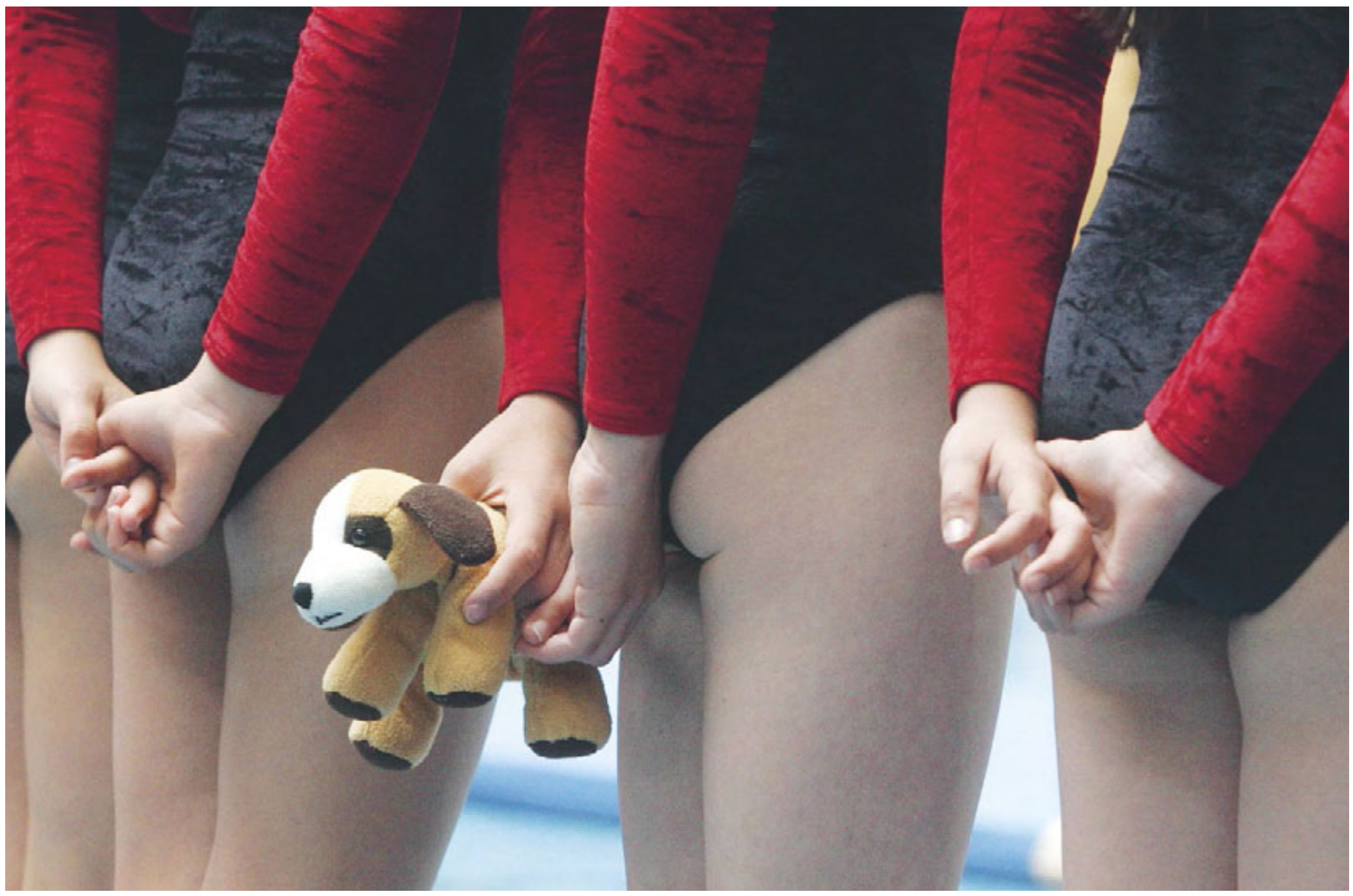

Gymnaste tenant un porte-bonheur lors d'une compétition de gymnastique, Mannheim, Allemagne, 16 juin 2005 (détail). ImageBROKER / Alamy Stock Photo.

autant du smartphone aujourd'hui pour les adultes et les adolescents, ou de nos ordinateurs). Mais quel être sensé dépenserait de l'argent pour un jouet sali, fatigué, usé? Deuxième pierre, done, dans le jardin des conceptions ordinaires et économistes du précieux: le fétiche est sans prix, non pas, comme l'objet de patrimoine, parce que sa valeur particulière l'a rendu inaliénable en le soustrayant au marché, mais parce que sa valeur objective, aux yeux de tout autre que son possesseur, est si faible qu'il n'est même pas susceptible de donner prise à l'échange marchand; seul l'usage fait sa force ${ }^{4}$.

\section{OBJETS-PERSONNES}

Quel est le point commun entre le fétiche (le doudou du bébé), la relique (la robe de Marilyn) et l'œuvre d'art (le tableau de Van Goghh)? C'est qu'ils sont insubstituables: une peluche quelconque ne peut pas remplacer celle qui s'est imprégnée de l'odeur du bébé, une copie même parfaite de la robe ne peut pas remplacer celle qui fut portée par Marilyn en personne, et un faux Van Goǵh, fût-il fidèle au point de tromper les meilleurs experts, ne sera plus qu'une croûte dès lors qu'il aura été repéré comme un faux. Fétiches, reliques et œuvres d'art sont ce que j'ai appelé des «objetspersonnes», qui partagent avec les humains (du moins les humains à l'état normal, non réduits en esclavage ni promis à extermination) cette propriété d'être inéchangeables au sens d'insubstituables, non susceptibles d'être remplacés par un autre objet (Heinich 1993). Les «objets-personnes», sous leur triple espèce du fétiche, de la relique et de l'œuvre d'art, sont des choses «en régime de singularité », c'est-à-dire traitées de façon à interdire toute équivalence avec une autre chose, soit parce qu'elles portent la trace d'une personne (la relique), soit parce qu'elles aǵissent comme une personne (le fétiche), soit les deux (l'œuvre d'art, qui porte la trace de son auteur et provoque des émotions sur le spectateur).

À la lumière de cette focalisation sur l'insubstituabilité des choses au même titre que des humains, la notion de "personne» se détache ainsi de la notion $\mathrm{d}^{\prime}$ «humain», pour devenir une «fonction» (analogue à la «fonction-auteur» décrite par Michel Foucault [1969]), affectée par principe aux humains et, dans certains cas, aux animaux ou aux choses ${ }^{5}$. Et c'est précisément cette propriété particulière qu'est l'insubstituabilité qui fait la préciosité, beaucoup plus qu'une valeur monétaire susceptible d'aller du néant (le doudou) à l'indéfini (le Van Gog̉h dans un musée).

C'est là une troisième pierre, donc, dans le jardin de l'économisme ordinaire: l'extension du cas du fétiche à sa catégorie de référence - l'objet-personne - oblige à relativiser la valeur qualifiante, si l'on peut dire, de la valeur marchande. Celle-ci n'est pas absente
4. Sur ces questions, voir notamment Appadurai 1988.

5. Il arrive toutefois,

exceptionnellement, que les humains soient traités comme des choses (substituables) et non comme des personnes (insubstituables), dans le cas des traitements dits, justement, «inhumains», propres à dégrader la personne dans sa dignité d'être humain (esclavage, déportation...). Mais ce sont, justement, des situations-limites, objets de fortes condamnations morales et juridiques. 
6. La notion d'«effet référentiel » a été développée dans Heinich 2012a.

7. Sur la capacité d'action des œuvres d'art, voir Gell 2009.

8. L'historien américain Patrick Geary a remarquablement décrit la circulation des reliques médiévales, entre don, vol ou commerce: en dépit de la constance de leuridentité , ces épreuves les font redécouvrir et changer de mode de présentation, de statut (de l'objet-personne ou de l'objet de patrimoine au déchet, en passant par le bien marchand) et bien sûr, de valeur (Geary 1988).
- comme dans le cas de l'objet de patrimoine, rendu inéchangeable au sens d'inaliénable, non susceptible de changer de mains -, mais elle devient non prédictible de la préciosité de l'objet: le prix n'est plus la bonne mesure de la valeur suprême.

Résumons l'état de la question à cette étape de notre réflexion: inaliénabilité (cas du patrimoine, du trésor) et insubstituabilité (cas des objets-personnes: fétiches, reliques, œuvres d'art) sont des propriétés fortement qualifiantes pour identifier le précieux, mais elles inversent la place de la qualification monétaire en la rendant soit non pertinente, soit si variable selon les contextes qu'elle en perd toute capacité à objectiver la valeur de l'objet. Bref : contrairement aux apparences, le coûteux n'est que margínalement la bonne mesure du précieux.

\section{MESURE, ATTACHEMENT, JUGEMENT}

Cette relativisation du prix comme mesure de la valeur du précieux nous amène à une deuxième extension: non plus seulement l'extension du précieux au-delà du coûteux mais aussi, plus généralement, l'extension de la valorisation au-delà de la mesure. Celle-ci en effet n'est qu'une forme, parmi d'autres, de l'attribution de valeur, qui peut prendre également deux autres formes tout aussi familières : la forme de l'attachement, et la forme du jugement de valeur.

Si la mesure s'exprime essentiellement par des chiffres (le prix), l'attachement se manifeste surtout par des actes, accompagnés ou non par des mots: le bébé s'accroche au doudou, le conservateur de musée surveille les œuvres laissées à sa garde et s'assure de leur sécurité, le collectionneur peine à prêter ses tableaux et se plaît à les montrer à ses amis, le fan de Johnny met sous verre l'autographe qu'il a réussi à lui soutirer, s'assurant ainsi qu'il sera à la fois protégé et exposé. Plus le soin est attentif, constant, organisé voire institutionnalisé, plus la préciosité est attestée, quelle que soit la valeur d'assurance qui aura été attribuée à l'objet. Il n'est que d'observer la minutie des manipulations auxquelles donne lieu le moindre déplacement d'un artefact dans un musée, les gants blancs des appariteurs, la choréǵraphie des mouvements parfaitement réglés, pour prendre la mesure de l'attachement dont cet artefact fait l'objet, c'est-à-dire de la valeur hors du commun qui lui est consensuellement accordée. Coûteux ou non, le précieux est d'abord ce dont on prend soin (Bonnot 2014; Dominguez Rubio 2016).

Enfin, la troisième forme d'attribution de valeur est le juǵement, l'énoncé par lequel s'exprime, plus ou moins directement, la grandeur d'un objet aux yeux de ceux pour qui il compte. De l'opinion énoncée en passant par l'homme ordinaire - «Moi, j'adore Rembrandt! » - au juggement d'expert longuement mûri, lourdement équipé, fortement contraint, juridiquement et économiquement performatif - «Je certifie que ce tableau est bien de la main de Rembrandt» -, les mots sont là pour produire des évaluations, que ce soit pour valoriser ou pour dévaloriser. «Trésor...», murmure une mère à son enfant, un amoureux à son amante, Harpagon à sa cassette, le curé à la relique qui fait la ǵloire de son église, l'athlète au grigriri qui l'aidera à gaǵner la compétition...

\section{LA MULTIPLICITÉ DES ÉTATS DE CHOSE}

Mesure, attachement, juǵement: ayant dû faire leur deuil d'une estimation chiffrée de la valeur de La Joconde, les visiteurs du Louvre manifestent leur attachement en la photographiant (ou en se photographiant avec elle), en achetant sa reproduction, en stationnant longuement devant elle, en quête probablement de l'expérience émotionnelle hors du commun que devrait logiquement provoquer la mise en présence avec un objet si précieux - cet «effet référentiel» qui se produit chaque fois qu'on se trouve en présence d'un être que l'on connaît déjà par sa reproduction, qu'il s'agisse d'une personne humaine ou d'un objet-personne $^{6}$. Et puis, ils en parlent, commentent, s'exclament: «Sublime», «Quelle merveille», «C'est si beau»...

Car La Joconde, œuvre d'art et objet de patrimoine, est aussi un fétiche: elle agit sur les gens, ne serait-ce qu'en les faisant aǵir - les incitant à venir du monde entier, à faire la queue, à acheter un billet, à passer du temps devant elle et pour elle. Il peut même arriver qu'elle agisse sur eux en provoquant une émotion, esthétique ou autre? ${ }^{7}$. Et puis, en tant qu'objet en trois dimensions, elle est aussi une relique, issue de la main d'un grand homme dont elle transporte un peu de la présence - l'artiste qui l'a peinte. OEuvre d'art patrimonialisée, donc non seulement insusbstituable mais aussi inaliénable, fétiche agissant sur des foules immenses et relique avérée par toute l'histoire de l'art, La Joconde est certainement le cas le plus emblématique et le plus connu d'objet-personne.

On voit là que la préciosité ne se manifeste pas seulement par la quantité d'argent, d'attachements ou de mots qui l'attestent: elle se déploie aussi sur l'éventail des états de la chose ainsi valorisée, de l'objet de patrimoine à l'objet de luxe et à l'objetpersonne, de l'œuvre d'art à la relique et au fétiche ${ }^{8}$. En d'autres termes, la multiplicité des états de la chose est, elle aussi, un indicateur de sa préciosité, c'est-à-dire, à la fois, une conséquence et une cause de l'extrême valeur qui lui est attribuée.

\section{CHOSES, PERSONNES, ACTIONS ÉTATS DU MONDE}

«États de la chose», donc: or là encore, il faut étendre le modèle. Car après l'extension du précieux au-delà du prix, l'extension de l'évaluation au-delà de la mesure, et l'extension de l'objet au-delà d'un unique état, il nous faut aussi accepter l'extension non plus des formes d'attribution de la valeur, mais de ses objets.

Les choses matérielles, en effet, ne sont pas les seuls «objets» d'attribution de valeur: nous passons beaucoup de temps à évaluer également les personnes, 
à évaluer leurs actions, et à évaluer les états du monde (par exemple lorsque nous valorisons la paix, la démocratie, etc., ou lorsque nous stiǵmatisons la violence ou le totalitarisme). Ainsi, certaines personnes sont précieuses non seulement pour leurs proches mais aussi pour l'ensemble d'une communauté, tels ces "grands singuliers» que sont les saints, les génies et les héros ${ }^{9}$. Certaines actions sont également dotées d'une valeur exceptionnelle, comme les records sportifs ou les actes d'héroïsme, dont les nations conservent précieusement le souvenir - par exemple, en France, l'appel du 18 juin 1940 du général De Gaulle. Enfin, certains états du monde sont jugés précieux par un grand nombre de personnes, prêtes à se battre pour conquérir ou conserver un État démocratique, une société laïque ou, au contraire, l'imposition à tous de rèǵles religieuses.

C'est dire une fois encore que pour produire une ontologie adéquate du précieux, il faut à la fois relativiser et étendre la conception ordinaire: l'étendre au-delà des choses, en direction des «objets » au sens abstrait de visées de la pensée ou de l'action; l'étendre au-delà du prix et de la mesure, en direction des attachements et des jugements ; et l'étendre, enfin, au-delà des biens, c'est-à-dire des objets valorisés à l'extrême, en direction, d'une part, des opérations qui conduisent à ces valorisations - on aura reconnu là le tournant praǵmatique - et, d'autre part, des principes au nom desquels ces valorisations sont produites et acceptées.

\section{GRANDEUR, BIENS, PRINCIPES}

Car si le «bien» précieux constitue une valeur suprême, c'est au deuxième sens du mot «valeur», selon la distinction des trois sens du mot «valeur» que j'ai identifiés dans Des valeurs (2017a). Au deuxième sens donc, une «valeur» est un objet auquel a été attribué de «la» valeur, au premier sens de «grrandeur» - devenant ce que nous nommons un «bien». Et pour que de «la»valeur» (premier sens) soit attribuée à un objet ainsi transformé en une "valeur» (deuxième sens), il faut que «des» valeurs, au troisième sens de principes d'évaluation, aient été projetées, plus ou moins consciemment, sur des objets, de façon à les transformer en «biens» (ou, à l'inverse, de façon à récuser leur statut de biens).

C'est dire que pour comprendre pleinement l'état de préciosité, il faut s'intéresser aux différentes opérations de production de cette valeur extrême (par le jugement, par les manifestations d'attachement, par la mesure), et à leurs soubassements que sont les représentations plus ou moins partagées des principes axiologiques susceptibles de justifier l'attribution de valeur.

C'est sur ce troisième et dernier sens du mot «valeur»- les principes organisant l'évaluation qu'il est temps de se pencher à présent pour pouvoir balayer l'ensemble de la "grammaire axiologique» qui commande l'imputation de préciosité.

\section{VALEURS-PRINCIPES}

Revenons au cas du doudou: pour que quelqu'un dépense de l'arǵent afin d'acquérir une peluche usagée, il faudrait que celle-ci, proposée sur le second marché - au pire un vide-greniers, au mieux un marché aux puces voire une salle des ventes - ait perdu son lien d'origine avec son premier possesseur et, si possible, ait gagóné en valeur d'ancienneté ce qu'il a perdu en valeur d'usage: un teddy-bear vintage, même et surtout s'il a servi, pourra dans ces conditions passer de l'état de fétiche à l'état d'objet de collection (Pomian 1987), susceptible d'appréciation monétaire (et il en va de même, bien sûr, du masque africain arraché à sa tribu d'origine pour être proposé aux amateurs du monde occidental [Derlon et Jeudy-Ballini 2008]).

Dès lors il ne sera pas inaliénable (sauf s'il entre dans les collections d'un musée), ni insubstituable (un autre masque semblable issu de la même tribu fera aussi bien l'affaire, de même qu'un autre teddy-bear issu de la même unité de fabrication); mais sa valeur particulière aux yeux du collectionneur - voire, éventuellement, sa préciosité - proviendra de l'association entre sa rareté et son ancienneté.

Ainsi, qu'un objet de collection soit d'autant plus précieux qu'il est rare et ancien nous oblige à prendre en compte, pour comprendre le statut de la préciosité, ces deux valeurs ajoutées, au sens où elles ne sont pas attribuables à l'état initial de l'objet: contrairement à la pierre précieuse, au bijou, au sac de luxe ${ }^{10}$, l'objet de collection n'a souvent été au départ qu'un vulgaire objet d'usage, à la matière triviale, au coût initial insignnifiant. C'est là le principe de la «mise en valeur» observée par Luc Boltanski et Arnaud Esquerre (2017) dans leur analyse de «l'enrichissement» - analyse qui aurait pu contribuer utilement à une sociologie du précieux s'ils n'avaient prétendu réduire la valeur à une simple stratégie de justification du prix ${ }^{11}$.

D'autres valeurs que le coût, la rareté et l'ancienneté sont susceptibles de rendre un objet précieux: notamment la beauté (celle des œuvres d'art comme celle des acteurs et des mannequins), le plaisir (celui que procure la drogue ou le mets raffiné), l'amour (celui qu'on nous porte, ou que nous portons à un être), l'authenticité (celle d'un tableau de maître), la virtuosité (celle d'une performance technique exceptionnelle), la célébrité (celle d'une star ou celle d'un tableau mondialement connu), la proximité (par l'appartenance à une même unité domestique), la significativité (le sens susceptible d'être contenu dans un objet, sa capacité à susciter un récit), la manifestation d'une transcendance (notamment par l'inspiration), mais aussi, plus trivialement, la fonctionnalité (robustesse, utilité). C'est ainsi qu'un grand nombre de 《registres de valeurs» (ou «familles» de valeurs ${ }^{12}$ ) sont impliqués dans la qualification du précieux: non seulement l'économique mais aussi l'esthétique, l'aesthésique, l'affectif, le pur, le technique, le réputationnel, le domestique, l'herméneutique, le mystique, le fonctionnel... Autant dire que la multiplicité des registres concernés fait écho à la richesse de l'objet précieux.
9. Pour un développement de cette triade empruntée à Max Scheler, voir Heinich 1991.

10. Sur le statut particulier du sac de luxe, voir Pillet-Anderlini 2016.

11. Pour un développement de cette critique, voir Heinich 2017b.

12. Selon le modèle des «ressemblances de famille» proposé par Ludwig Wittgenstein dans Recherches philosophiques, Paris, Gallimard, 2005 [1953]: 64. 


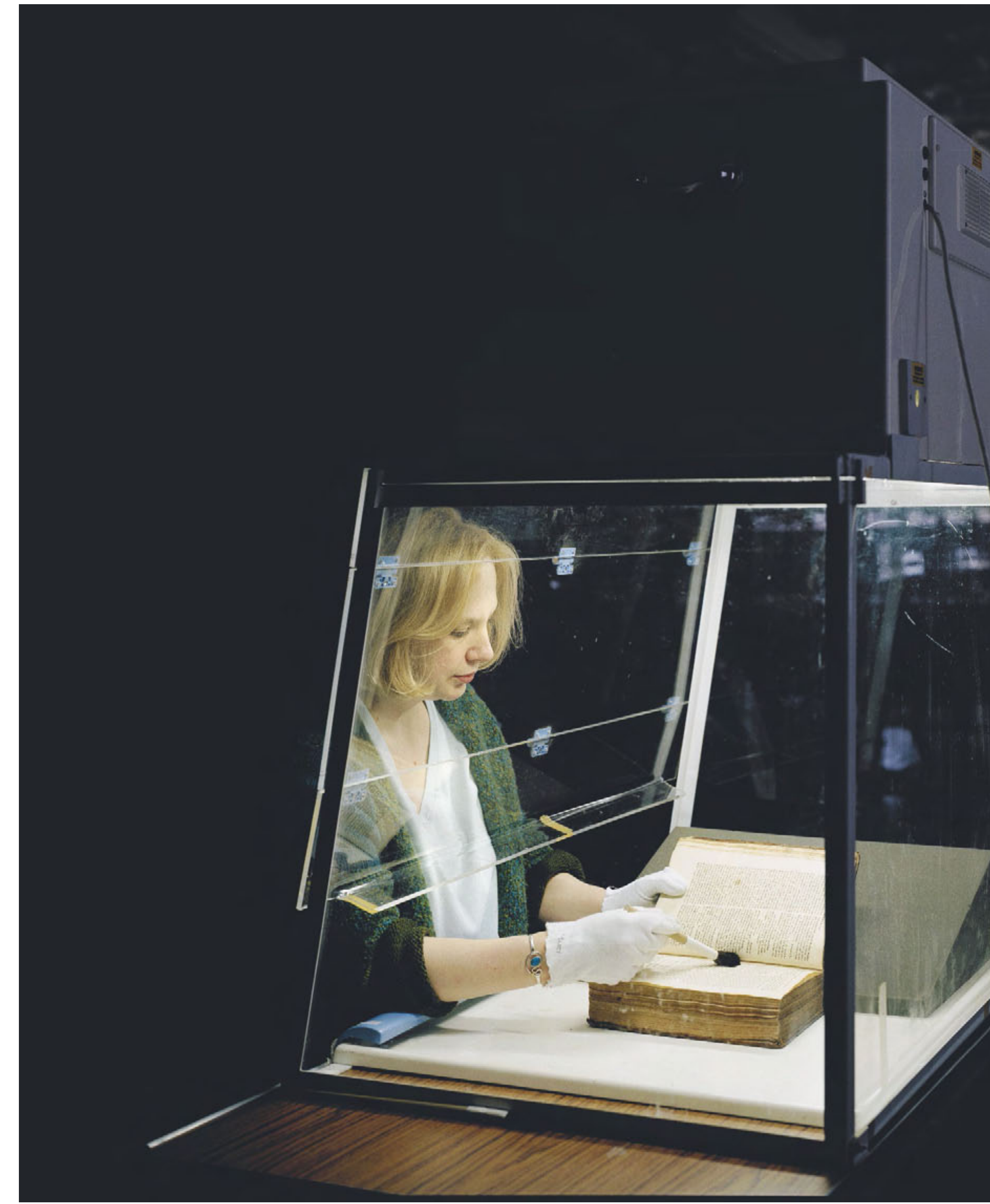


Mark Power, Les œuvres du National Trust sont affectées par les changements climatiques. Une restauratrice au Blickling Hall nettoie des ouvrages anciens abîmes par des insectes.

Norfolk, Grande-Bretagne, mai 2006 @ Mark Power/Magnum Photos. 
Dossier

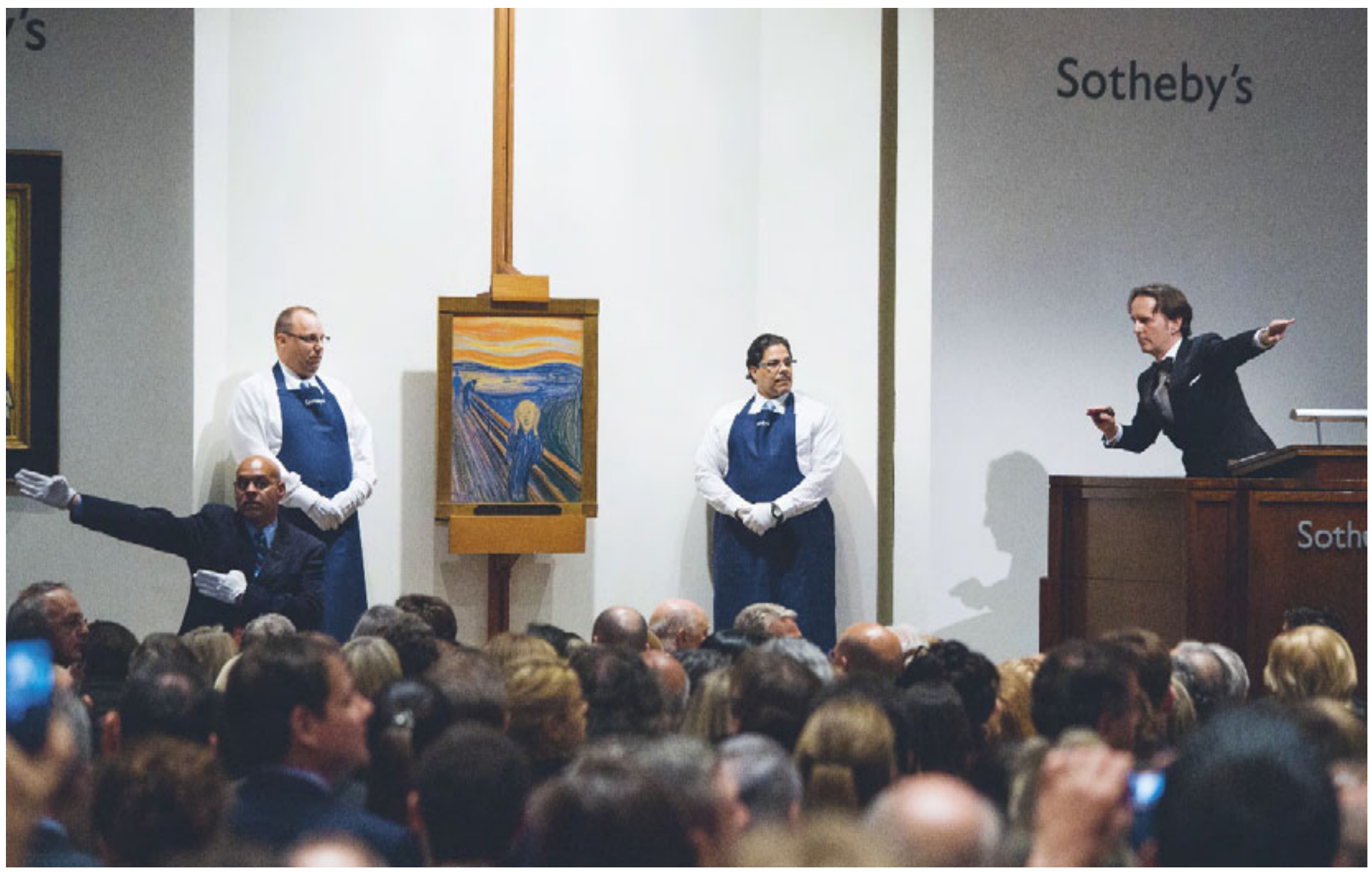

Orjan F. Ellingvag, Vente d'une des cinq versions du Cri d'Edvard Munch, 2 mai 2012. La vente est menée par le commissaire-priseur Tobias Meyer. Photo d'Orjan F. Ellingvag/Corbis via Getty Images. 
Une grande beauté, une grande capacité à procurer du plaisir ou à inspirer de l'amour, la mise en présence de l'objet authentique ou d'une célébrité dont l'image nous est familière ont la propriété de provoquer en nous des émotions - et des émotions positives, contrairement à l'indignation, qui signale le sentiment qu'une valeur a été transǵressée. L'émotion est bien un indicateur de valeur: négative dans le cas où une valeur n'a pas été respectée, et positive dans le cas contraire, lorsqu'une valeur est fortement implémentée dans une chose, une personne, une action, un état du monde. Plus l'objet en question est précieux, plus l'émotion est puissante: l'émotion est, si l'on peut dire, un signal de préciosité.

\section{AMPLIFICATEURS \\ DE VALEURS ET RÉGIMES DE QUALIFICATION}

Nous avons vu précédemment, avec la rareté et l'ancienneté, des valeurs au statut un peu particulier, en ce sens qu'elles peuvent s'ajouter à n'importe quelle autre valeur pour en amplifier la force: ce pour quoi elles n'appartiennent pas à un «registre de valeurs » en particulier, mais peuvent s'appliquer à tous. C'est le cas également de l'universalité et de la nouveauté qui, comme la pérennité (ou l'ancienneté) et la rareté, s'ajoutent à la beauté d'une œuvre d'art ou d'un objet de patrimoine pour en faire une œuvre plus précieuse qu'une autre. Rareté et universalité sont des amplificateurs dans l'espace, nouveauté et pérennité sont des amplificateurs dans le temps.

Reprenons le cas, décidément emblématique, de La Joconde : elle est rare bien sûr, puisque insubstituable - et les rumeurs récurrentes selon lesquelles l'œuvre exposée est une copie, l'original étant conservé à l'abri des regards, montre bien a contrario l'importance de cette attente d'insubstituabilité, stade suprême de la rareté puisqu'elle implique l'unicité. En même temps, l'œuvre est universelle par la multiplicité de ses admirateurs, dans le monde entier: loin de ne concerner que les Italiens, les Français, ou même les Occidentaux, elle fascine jusqu'en Extrême-Orient. Par ailleurs, elle est pérenne: non seulement elle est ancienne, mais elle est présumée devoir durer très longtemps pour être transmise aux générations futures, et tout est fait pour lui assurer cette pérennité maximale, indissociable de son statut patrimonial. Quant à son originalité ou à sa nouveauté, il faut pour l'établir rapporter l'œuvre à celles qui lui furent contemporaines, et mettre en avant un certain nombre de critères permettant d'établir en quoi elle n'est pas le simple élément d'une série, mais une création hors du commun, due à un artiste lui-même exceptionnel, et dotée par conséquent d'une réelle singularité, au sens fort - c'est-à-dire non seulement une spécificité, mais une exceptionnalité.

L'on constate ainsi que ces quatre amplificateurs de valeur, ou «valeurs cardinales », se déploient selon le double axe de l'espace (universalité, rareté) et du temps (pérennité, nouveauté). Mais ils se déploient aussi selon l'opposition entre deux grands régimes de qualification, à savoir le «régime de communauté » (la valeur de principe accordée au commun, au partagé, au conventionnel, au multiple) et le «régime de singularité» (la valeur de principe accordée à l'exceptionnel, au rare, à l'unique, au nouveau) ${ }^{13}$. Ainsi l'universalité et la pérennité sont des amplificateurs en régime de communauté - dans l'espace pour l'une, dans le temps pour l'autre -, tandis que la rareté et la nouveauté sont des amplificateurs en régime de singularité, dans l'espace et dans le temps. Toucher le plus grand nombre, traverser les générations et les siècles sont des exigences propres à satisfaire les attentes d'un monde commun, relevant du «régime de communauté»; tandis qu'être une œuvre à nulle autre pareille, avoir ouvert en son temps des voies créatives inédites sont des exiǵences propres à satisfaire les attentes d'exceptionnalité, relevant du «régime de singularité». Ce sont là deux façons antinomiques, mais tout aussi efficaces l'une que l'autre selon les contextes, de construire une relation d'admiration envers un objet ou un être humain - et d'admiration partageable, et partagée.

La Joconde, pour revenir à cet exemple emblématique, peut done voir sa valeur amplifiée et dans le temps, et dans l'espace, et dans les deux régimes de qualification - communauté et singularité. Voilà donc une autre façon de construire la «mise en valeur» du précieux: non plus à travers l'indicateur de l'émotion constatée, mais à travers la décomposition analytique des «valeurs»- au sens de principes axiologiques susceptibles de qualifier un objet. C'est ainsi que la "ǵrammaire axiologique» proposée dans Des valeurs (2017a) nous permet de mieux comprendre de quoi est fait le précieux.

\section{ENTRE LE DÉGHET ET LE SACRÉ}

Nous pouvons à présent positionner le précieux à l'extrémité d'un axe de valorisation, dont l'extrémité opposée est le déchet ${ }^{14}$ : le précieux, c'est d'abord ce qu'aucun membre d'une communauté ne songerait à jeter, hier, aujourd'hui ou demain.

Les interdits portant sur une eatégorie d'objets sont d'ailleurs, comme les émotions, un autre indicateur de préciosité: interdit de jeter, mais aussi interdit d'échanger, interdit de substituer et même, dans certains cas, interdit de montrer. L'on pourrait d'ailleurs ordonner les différents types de précieux selon une gradation ordonnée en fonction de ce paramètre: au plus bas niveau, le précieux domestique, concernant les objets d'usage considérés comme suffisamment utiles pour qu'on les conserve; puis, les objets irremplaçables, appartenant à une catégorie considérée comme nécessaire (la poêle à frire dans une batterie de cuisine, qu'on ne peut remplacer par une casserole, et réciproquement; ou encore le bijou de famille, qu'on ne peut vendre ${ }^{15}$ ); à un degré supérieur encore, les objets-personnes, considérés comme insubstituables au moins par leur possesseur (tel le bébé tenant à son doudou), voire par leurs conservateurs désignés par la collectivité (tel le gardien des reliques, des fétiches, des œuvres de musée);
13. Pour une présentation détaillée et exemplifiée de ces deux régimes de qualification, voir Heinich 1991 et 2005.

14. À ce sujet, voir notamment Thompson 1979; Debary 2019.

15. Lire à ce sujet la

remarquable analyse par Thierry Bonnot (2006) des différents états d'un bijou décrits dans un célèbre roman. 


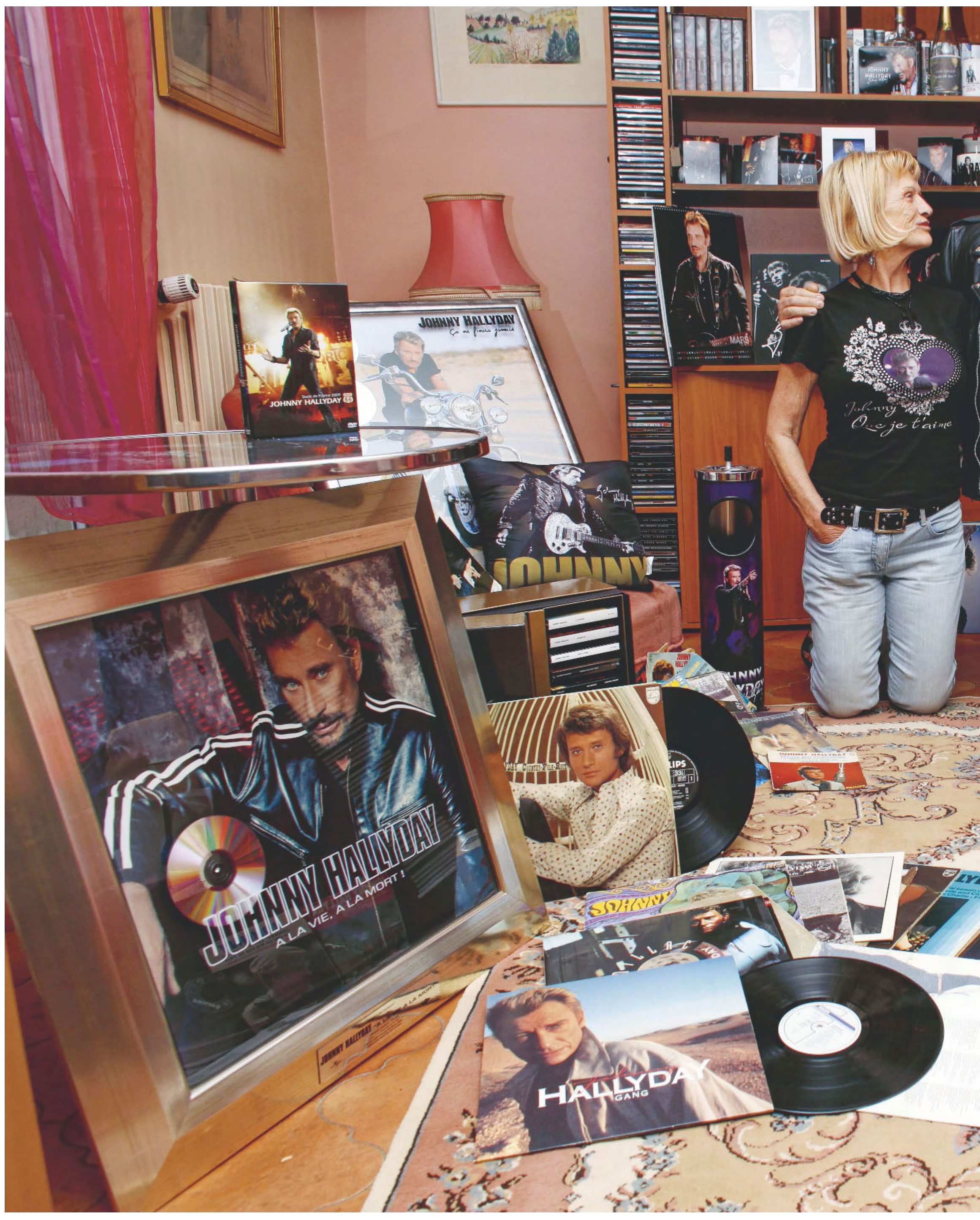




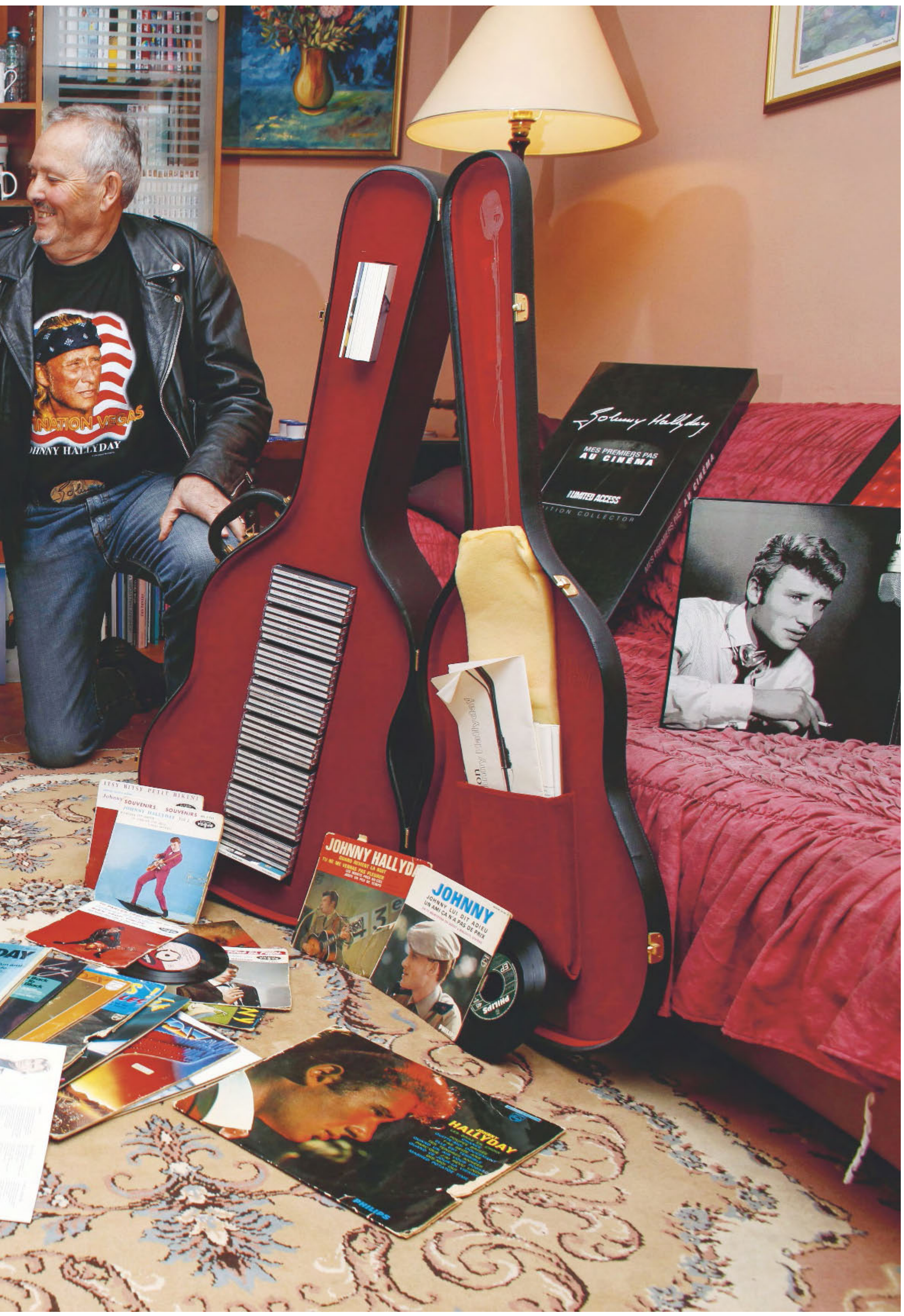


16. Pour d'autres exemples d'«empêcheurs de penser», voir Heinich 2009b.

17. J'ai développé ce point dans Heinich 2012b; 2017c.

18. Voir Mauss 1950. enfin, les objets dotés d'un statut suffisamment sacralisé pour instaurer une séparation à l'intérieur de la communauté, entre les membres autorisés à les approcher et ceux à qui il est interdit de les toucher, voire de les regarder.

Cette introduction, dans notre problématique, de la problématique du «sacré» nous amène à poser, pour finir, la question suivante: que gagnerait-on à remplacer la notion de précieux par celle de sacré? Certes, cette substitution a du sens pour peu que l'on définisse le sacré, comme nous venons de le faire à propos du précieux, comme la forme suprême de valorisation. Il y aurait ainsi, au sommet, la sacralisation (ou, sous sa forme laïque, la patrimonialisation); puis des étapes intermédiaires faisant intervenir, selon, l'appréciation monétaire (registre économique), l'interprétation (registre herméneutique), l'amour (registre affectif); plus bas, l'invisibilité, en ce qui concerne la non-valeur; puis la dévalorisation, en cas de jugement de valeur négatif; et enfin, tout en bas, la dépouille ou le déchet, objets de rejet.

Si donc le sacré se situe, avec le précieux, au sommet de cette chaîne d'étapes axiologiques, si done ces deux termes sont équivalents, pourquoi parler de «sacré» plutôt que de «précieux »?

Il y aurait une justification sans appel à ce choix: ce serait au cas où les acteurs eux-mêmes emploieraient le terme. Cela peut arriver, certes, mais seulement dans ces contextes bien particuliers que sont les contextes religiieux ou maǵiques. En dehors de ces arènes relativement bien repérées, le terme de «sacré» n'est utilisé, semble-t-il, que dans des textes savants, et de façon métaphorique: l'art comme forme modernisée du «sacré», le «sacre» des ǵrands sportifs, la «sacralisation» du chef politique, voire - comme dans un récent ouvrage de sociologie - le «sacré» comme forme cachée de «domination», etc.; ou encore, comme ici, le précieux comme forme du «sacré » (à moins qu'il s'agisse du «sacré» comme forme du précieux?). Dans ces conditions, il vaut la peine de se demander ce qu'on gaǵne, et ce qu'on perd, à une telle extension, propre au monde savant, de la catégorie du «sacré» au-delà de sa sphère spécifiquement reliǵieuse.

Ce qu'on y gagne, c'est probablement l'impression de mieux comprendre notre objet, parce que le «sacré», terme familier, nous paraît du même coup éclairant. Or je pense qu'il fonctionne plutôt comme un «empêcheur de penser ${ }^{16} »$, une notion qui barre la réflexion en la faisant buter sur un interprétant propre à nous donner le sentiment d'un gain d'intelligence. Mais qu'y gagnons-nous effectivement, que comprenons-nous exactement que nous n'aurions pas compris sinon? Je ne le vois pas bien.

Je vois en revanche deux inconvénients à cette mise en équivalence du précieux et du sacré. Le premier, c'est d'inciter à raisonner en termes de valorisation décontextualisée, sans limitations spatiales ou temporelles, donc absolue: le sacré serait sacré «en soi», universellement, intemporellement, ainsi que le voient les acteurs. La pente est ǵlissante en effet entre une définition ontologique - ce qui caractérise une entité dans l'expérience des acteurs - et une définition métaphysique ou essentialiste - ce qu'est une entité «en soi», indépendamment de l'expérience. En d'autres termes, la pente est ǵlissante - nous le savons tous - entre la réflexion sur les catégories utilisées par les acteurs, et la production de catégories pour les acteurs. Hormis, encore une fois, lorsqu'il s'agit d'analyser ce que les acteurs entendent par là lorsqu'ils emploient ce terme, ou ses équivalents, je crains que le passage par la notion de «sacré» ne relève la plupart du temps de cette seconde conception, assez peu scientifique me semblet-il, du métier de chercheur en sciences sociales, conçu moins comme producteur de savoir que comme pourvoyeur de leçons aux acteurs quant à ce qu'ils devraient penser.

Le second inconvénient que je vois à l'invocation du «sacré» comme équivalent du précieux, c'est d'enfermer l'une et l'autre notions dans une ganǵue reliǵieuse. Ce faisant, l'on s'interdit de décrire les passages d'une arène à une autre, les déplacements de valorisations entre objets hétérogènes, ainsi que les points communs entre ces entités, au-delà des variations. Car de deux choses l'une: ou bien la sacralisation appartient en droit au domaine religieux, de sorte que toutes ses formes dérivées, dont le précieux, seraient des ersatz impurs de religiosité - ce qui revient à faire de la reliǵion une matrice explicative, universelle et intemporelle, c'est-à-dire une réalité métaphysique. Ou bien la sacralisation religieuse n'est qu'une des formes de sacralisation, définie comme une forme supérieure de valorisation; mais alors, pourquoi mettre au premier plan de l'analyse une catégorie - le sacré - qui n'en est qu'une modalité parmi d'autres?

On aura compris que c'est cette seconde position - éviter de référer a priori à une catégorie religieuse - qui me paraît légitime, parce que conforme au programme épistémologique des sciences humaines et sociales. Non seulement nous n'avons nul besoin du «sacré » pour comprendre nos objets - ce sont les acteurs, et non les chercheurs, qui peuvent en avoir l'usage - mais, pire, ce terme risque de bloquer notre effort de réflexion et de nous enfermer dans une matrice explicative générale qui n'est, de fait, ni si générale, ni si explicative, ni si matricielle qu'elle n'en a l'air ${ }^{17}$.

Dans ces conditions, il me paraît beaucoup plus heuristique de considérer le précieux non pas comme une propriété constitutive qui expliquerait les opérations de valorisation et de singularisation (où l'on retrouve la croyance des acteurs dans la transcendance du «sacré»), mais bien plutôt comme le produit de ces opérations. Et après tout, nous ne ferons ainsi que retrouver le geste conceptuel de Marcel Mauss inversant la cause et l'effet du don, lequel n'est plus, dans son analyse, l'effet du «Hau» contenu dans l'objet, mais la cause du statut particulier affecté à cet objet ${ }^{18}$. De la même façon, la valeur d'un objet n'est pas la cause mais la conséquence des opérations qui font de cet objet un objet de valeur - fût-ce un objet de très grande valeur, tel un objet précieux.

CNRS-EHESS nathalie.heinich@ehess.fr 
Axiologie du précieux. Essai de modélisation.

Nathalie Heinich

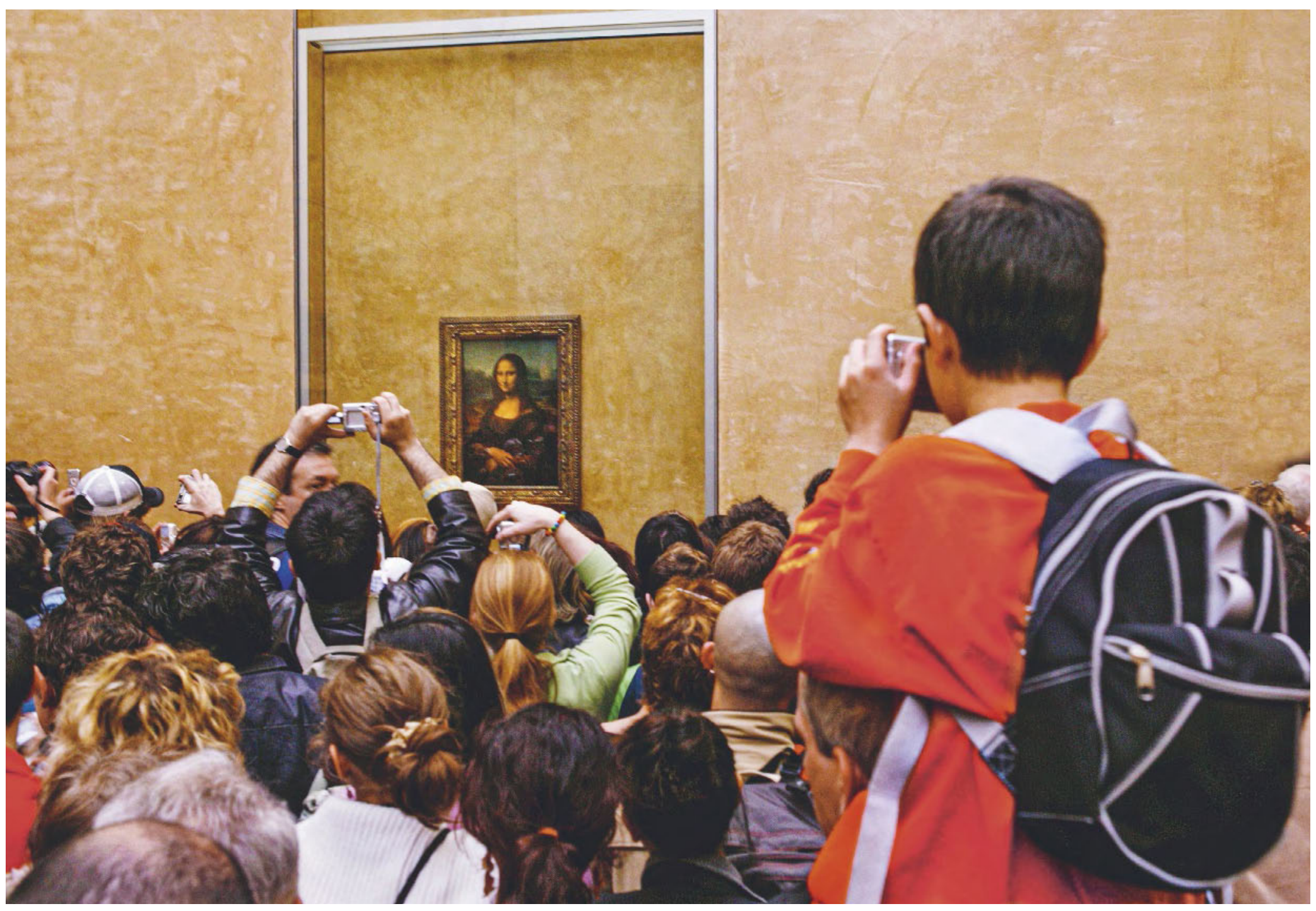

Raphaël Gaillarde, Ouverture de la nouvelle présentation de La Joconde de Léonard de Vinci dans la Salle des États, musée du Louvre, 6 avril 2005 c) Raphaël GAILLARDE / Gamma-Rapho. 


\section{Bibliographie}

Appadurai, Arjun (dir.)

1988 The Social Life of Things: Commodities in Cultural Perspective. Cambridge/New York/New Rochelle, Cambridge University Press.

\section{Boltanski, Luc} et Esquerre, Arnaud

2017 Enrichissement: une critique de la marchandise. Paris, Gallimard.

\section{Bonnot, Thierry}

2006 «Qu'est-ce qu'un objet précieux? Au sujet d'un roman de Louise de Vilmorin ", Ethnologie française 36 '(4): 723-733.

2014 L'Attachement aux choses. Paris, CNRS Éditions.

\section{Debary, Octave}

2019 De la poubelle au musée. anthropologie des restes. Paris, Creaphis

\section{Derlon, Brigitte et} Jeudy-Ballini, Monique

2008 La Passion de l'art primitif: enquête sur les collectionneurs. Paris, Gallimard.

Domínguez Rubio, Fernando 2016 «On the Discrepancy Between Objects and Things An Ecological Approach », Journal of Material Culture 21 (1) : 59-86.

\section{Geary, Patrick}

1988 «Sacred Commodities: the Circulation of Medieval Relics », in Arjun Appadurai, The Social Life of Things: Commodities in Cultural Perspective. Cambridge, Cambridge University Press: 169-192.

\section{Gell, Alfred}

2009 L'Art et ses agents : une théorie anthropologique, trad. de l'anglais par Sophie et Olivier Renaut. Dijon, Presses du réel.

\section{Godelier, Maurice}

1996 L'Énigme du don. Paris, Fayard.

\section{Foucault, Michel}

1969 «Qu'est-ce qu'un auteur?", Bulletin de la société française de philosophie 63

(3) : 73-104.

\section{Heinich, Nathalie}

1991 La Gloire de Van Gogh: essai d'anthropologie de l'admiration. Paris, Minuit.

1993 «Les objets-personnes. Fétiches, reliques et œuvres d'art », Sociologie de l'art 6 . 25-55, repris dans Nathalie Heinich et Jean-Marie Schaeffer, Art, création, fiction: entre sociologie et philosophie.

Nîmes, Jacqueline Chambon, 2004 .
2005 L'Élite artiste :

excellence et singularité en régime démocratique. Paris, Gallimard.

2009a La Fabrique du patrimoine: de la cathédrale à la petite cuillère. Paris, Maison des sciences de l'homme. 2009b Le Bêtisier du sociologue. Paris, Klincksieck.

2012a De la visibilité: excellence et singularité en régime médiatique. Paris, Gallimard.

2012b «Des limites del'analogie reliǵieuse : l'exemple de la célébrité», Archives de sciences sociales des religions 158 :

157-177.

2017a Des valeurs: une approche sociologique. Paris, Gallimard.

2017b «Misères de la sociologie critique», Le Débat 197 : 119-126. 2017c «Pour en finir avec "le religieux": vers une analyse fonctionnelle des religiosités actuelles », Interrogations? [en ligne] disponible sur: https://www. revue-interrogations.orǵ/ Pour-en-finir-avec-le-reliǵieux

\section{Mauss, Marcel}

1950 "Essai sur le don», Sociologie et anthropologie. Paris, PUF : 143-279.

\section{Pillet-Anderlini, Véronique}

2016 «L'artification du luxe dans le discours médiatique: converǵence des siónes et du sens ». Thèse de doctorat en sciences de l'information et de la communication, université de Nice-SophiaAntipolis, sous la direction de Nicolas Pélissier.

\section{Pomian, Krzysztof}

1987 Collectionneurs, amateurs et curieux: Paris,

Venise, $X^{e}{ }^{e}$-XVIII ${ }^{e}$ siècle.

Paris, Gallimard.

\section{Thompson, Michael}

1979 Rubbish Theory:

The Creation and Destruction of Value. Oxford, Oxford University Press.

\section{Ci-contre et ouverturee}

Orjan F. Ellingvag, Vente d'une des cinq versions du Cri d'Edvard Munch, 2 mai 2012 (détail). La vente est menée par le commissairepriseur Tobias Meyer. priseur Tobias Meyer. Photo
d'Orjan F. Ellingvag/Corbis via Getty Images. 


\section{Sotheby's}
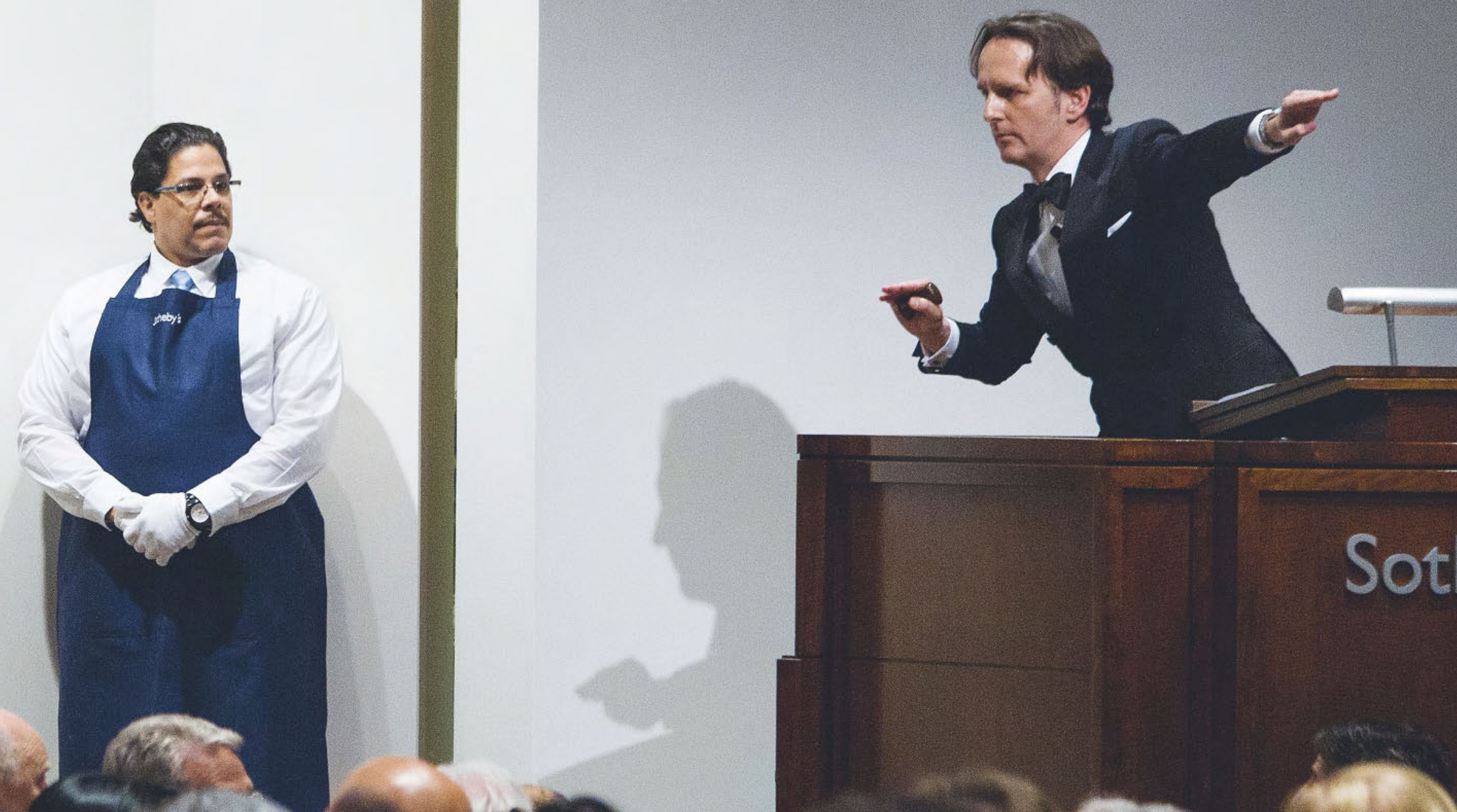